EUROPEAN ORGANIZATION FOR NUCLEAR RESEARCH

Laboratory for Particle Physics

Divisional Report

CERN LHC/2001-1 (MTA)

CERN-NuFact-Note 065

\title{
A Stationary Target for the CERN-Neutrino-Factory
}

\author{
Peter Sievers
}

As production target for Neutrino Factories, free mercury jets with high axial velocity of about $20 \mathrm{~m} / \mathrm{s}$ are being studied. For the CERN-Neutrino-Factory proposal with a 4 MW beam power, but with a relatively large beam size at $2.2 \mathrm{GeV} / \mathrm{c}$ and pulsed at $75 \mathrm{~Hz}$, maximum energy deposition densities of below $20 \mathrm{~J} / \mathrm{g}$ and average power densities of about $1 \mathrm{~kW} / \mathrm{g}$ are expected. Therefore a study has been made which discusses the feasibility and limits of a confined, stationary target cooled by a liquid. It is proposed to use solid spheres of high density material with diameters in the millimeter range. These spheres are confined inside a Titanium container and cooled by an efficient water circuit. Alternatively, low density liquid metal cooling could be used. Dynamic response, as pressure pulses and vibrations are greatly reduced by the small size of the target granules in combination with a relatively long beam pulse with a duration of $3.3 \mu \mathrm{s}$. The open issue of the lifetime of such as structure and its fatigue limit at a rate of 6.5 Mio. cycles per day must be assessed experimentally. Efforts should be invested in devising laboratory experiments simulating the beam conditions to optimize the design and to elucidate the limiting factors of such a target.

LHC Division

Administrative Secretariat LHC Division CERN

CH - 1211 Geneva 23
Geneva, Switzerland

8 March 2001 


\section{Introduction}

For the Neutrino-Factory, presently being studied at CERN [Ref. 1] an average beam power of $4 \mathrm{MW}$, incident on the production target is considered. The beam is pulsed at $75 \mathrm{~Hz}$, each pulse contains a bunch train with a duration of $3.3 \mu$ s and the pulse separation is $13.3 \mathrm{~ms}$. With $1.5 \times 10^{14}$ protons per pulse at a momentum of $2.2 \mathrm{GeV} / \mathrm{c}$ each beam pulse contains an energy of $53.3 \mathrm{~kJ}$. From MARS-cascade simulations [Ref. 2, 3] with a Gaussian radial profile with a r.m.s. radius of $1 \mathrm{~cm}$ of the incident proton beam, the peak energy deposition $\mathrm{q}_{0}$ along the axis of a mercury target is about 14.5 J/g, pulse [Ref. 2]. The radial energy distribution in the target is also about Gaussian with a r.m.s. radius w of $1.15 \mathrm{~cm}$, slightly larger than the r.m.s. radius of the proton beam. The energy density distribution varies only slightly along the target. With these input parameters and in particular, with the radial energy deposition density

$$
q(r)=\mathrm{q}_{0} \mathrm{e}^{-r^{2} / 2 \mathrm{w}^{2}}[\mathrm{~J} / \mathrm{g}, \text { pulse }]
$$

one can assess the feasibility to evacuate the energy from a solid stationary target by cooling it with a fluid. For this assessment we can reasonably assume that the energy deposition density for materials with different densities than mercury will remain about the same.

To evacuate from a solid cylinder (or a rod or sphere) an uniformly deposited power, the distance $\mathrm{R}$ from its center to its cooled surface must be kept as small as possible since the peak temperature in the center is proportional to $\mathrm{R}^{2}$. Moreover, the surface through which the heat is evacuated into the fluid must be maximized.

Evidently, a spherical geometry is optimal for this. Therefore in the following we consider a target made out of small spheres (pellets) which are encapsulated in a pipe, capped at both ends with windows. The cooling is ensured by flowing a liquid through the spaces between the spheres. The principle is shown in Fig. 1.

The effective pion and finally the neutrino yield which can be expected from the considered, rather wide beam and target diameters is clearly a subject which depends strongly on the production and absorption cross-sections of the wide spectrum of pion momenta and on the collection channel. The assessment of these issues, as yet rather undefined, is outside the scope of this study. 


\section{Water Cooled Spheres}

\subsection{The spheres}

To intercept all of the incident protons and to contain most of the cascade energy a target radius of $2.25 \mathrm{~cm}$ is considered. For the size of the target spheres we assume at most $3 \mathrm{~mm}$, while the minimum size may go down to $1 \mathrm{~mm}$. For the sake of the following arguments we take a radius $\mathrm{R}$ of $1 \mathrm{~mm}$ and a " packing factor " (i.e. volume of spheres relative to total volume) of $60 \%$. In order to come close to a target with an effective density of mercury $\left(\rho=13.5 \mathrm{~g} / \mathrm{cm}^{3}\right)$ we consider Tantalum spheres with a density of $\rho=16.8 \mathrm{~g} / \mathrm{cm}^{3}$ which results in an effective density of

$$
\bar{\rho} \approx 10 \mathrm{~g} / \mathrm{cm}^{3} .
$$

This leads to about 140 spheres $/ \mathrm{cm}^{3}$ and the surface per unit volume available for cooling is

$$
\varepsilon=18 \mathrm{~cm}^{2} / \mathrm{cm}^{3} \text {. }
$$

Evidently, other heavy materials like Tungsten alloys or Tungsten Carbide may be considered. But to arrive at a consistent set of parameters, in the following only Tantalum is assumed.

\subsection{Water cooling circuit}

To keep the peak and average temperatures of the spheres as low as possible, a high velocity, high pressure water circuit is considered. As indicated in Fig. 1 the water is passing in radial direction through the target in order to maximize the total flow and to reduce the transit time of the water. The effective free cross-section available for the water relative to the total cross-section is about

$$
\mathrm{f}=20 \% \text {. }
$$

Therefore, with a water velocity of

$$
\mathrm{v}=6 \mathrm{~m} / \mathrm{s}
$$

between the spheres, a water flow of

$$
\mathrm{V}=11 \mathrm{l} / \mathrm{s}
$$

is required for a target length of $\mathrm{L}=18 \mathrm{~cm}$.

Since an average power of $800 \mathrm{~kW}$ [Ref. 2], about $20 \%$ of the beam power is deposited in the target the average temperature rise in the cooling water is about $18 \mathrm{~K}$. 
For the water inlets and outlets arrays of pipes (9 pipes along each of the four arrays, see Fig. 1) with an inside and outside diameter of $1.2 \mathrm{~cm}$ and $1.4 \mathrm{~cm}$ respectively ensure an average water velocity in the inlets and outlets of also about $6 \mathrm{~m} / \mathrm{s}$. These pipes may be shaped and arranged to minimize the loss of pions emerging from the target.

With the above conditions for the water circuit a Reynolds number $R_{e} \approx 10^{4}$ results inside the target which is well in the turbulent regime, but still reasonably conservative. From this a heat transfer coefficient $\gamma$ of

$$
\gamma \approx 22 \mathrm{~kW} / \mathrm{m}^{2} \mathrm{~K}
$$

should be obtained. High efficiency heat exchangers, as developed by K. Schubert et al. [Ref. 4] have achieved similar values.

The pressure required to push the water at the velocity of $6 \mathrm{~m} / \mathrm{s}$ radially through the target is about $400 \mathrm{kPa}$ (4 bars).

High water speed is not only required for an elevated heat transfer coefficient but also to minimize the exit temperature of the water. The average temperature increase $\overline{\Delta T}$ is only about $18 \mathrm{~K}$. However, the water stream passing through the center of the target where the highest beam energy is deposited may exit locally at twice the average temperature:

$$
\Delta \mathrm{T} \approx 36 \mathrm{~K} .
$$

\subsection{Temperature of the spheres}

Instead of considering merely average power depositions and steady state temperatures, valid for an equivalent continuous proton beam, we try to assess the pulsed nature of the thermal regime.

As stated in paragraph 2.2 the average temperature rise in the water is relatively low so that we can assume an exponential decay of the temperature of each sphere after it has been heated instantaneously by $\Delta \mathrm{T}_{0}$. The time constant $\mathrm{t}_{0}$ of this exponential decay is given by

$$
\mathrm{t}_{0}=\frac{\mathrm{cm}}{\gamma \mathrm{F}}
$$


c: Specific heat of the sphere, $151 \mathrm{~J} / \mathrm{kg} \mathrm{K}$ for Ta

$\mathrm{m}$ : Mass of the sphere, $70 \mathrm{mg}$ for $\mathrm{R}=1 \mathrm{~mm}$ for $\mathrm{Ta}$

F : Surface of the sphere, $12.6 \mathrm{~mm}^{2}$ for $\mathrm{R}=1 \mathrm{~mm}$

$\gamma$ : Heat transfer coefficient, $22 \mathrm{~kW} / \mathrm{m}^{2} \mathrm{~K}$.

This yields

$$
\mathrm{t}_{0}=38 \mathrm{~ms} \text {. }
$$

From the condition of a stable thermal cycling, in phase with the beam pulses, we get:

$$
\left(\mathrm{T}_{1}+\Delta \mathrm{T}_{0}\right) \mathrm{e}^{-\mathrm{t}_{1} / \mathrm{t}} 0=\mathrm{T}_{1}
$$

$\mathrm{T}_{1}$ : Temperature just before the proton pulse

$\Delta \mathrm{T}_{0}$ : Instantaneous temperature rise caused by each proton pulse

$\mathrm{t}_{0}: 38 \mathrm{~ms}$

$\mathrm{t}_{1}: \quad 13.3 \mathrm{~ms}$, time between two beam pulses

which yields

$$
\mathrm{T}_{1}=2.3 \Delta \mathrm{T}_{0}
$$

Assuming as inlet temperature of the cooling water close to zero centigrade and limiting the maximum peak temperature of the hottest sphere to the boiling point of water of $100^{\circ} \mathrm{C}$, it results

$$
\begin{aligned}
& \mathrm{T}_{1}=70^{\circ} \mathrm{C} \\
& \Delta \mathrm{T}_{0}=30 \mathrm{~K} .
\end{aligned}
$$

This temperature limit would only allow for a maximum energy deposition density of

$$
\mathrm{q}=4.5 \mathrm{~J} / \mathrm{g}
$$

which is a factor of 3 lower than required.

Film boiling on the surface of the hottest sphere could lead to decreased heat transfer and run away. Therefore, to work at peak temperatures above $100{ }^{\circ} \mathrm{C}$ one may use a water cooling circuit, pressurized at the outlet to 5 bars which would raise the boiling point to about $150{ }^{\circ} \mathrm{C}$. 
For this case it is

$$
\begin{aligned}
& \mathrm{T}_{1} \approx 100^{\circ} \mathrm{C} \\
& \Delta \mathrm{T}_{0} \approx 50 \mathrm{~K} .
\end{aligned}
$$

Therefore a peak energy deposition density of $\mathrm{q}_{0}=7.5 \mathrm{~J} / \mathrm{g}$, half of the design value could be sustained in a pressurized water circuit.

Finally, as the time constant $t_{0}$ increases proportional to the radius of the sphere, with smaller spheres of $\mathrm{R}=0.5 \mathrm{~mm}$ and thus $\mathrm{t}_{0}=19 \mathrm{~ms}$, one achieves with the pressurized water circuit

$$
\begin{aligned}
& \mathrm{T}_{1}=75^{\circ} \mathrm{C} \\
& \Delta \mathrm{T}_{0}=75 \mathrm{~K} \\
& \mathrm{q}_{0}=11.3 \mathrm{~J} / \mathrm{g} .
\end{aligned}
$$

This is already $78 \%$ of the design value, allowing for an incident beam power of $3 \mathrm{MW}$. The absolute peak temperature of the " hottest " sphere limited to $150{ }^{\circ} \mathrm{C}$ may still provide some margin since the energy per pulse from one sphere will just be enough to boil off over $13.3 \mathrm{~ms}$ a water layer with a thickness of about $0.1 \mathrm{~mm}$ around the sphere. This is due to the high boiling heat of water. One may expect that this boiling layer will not fully develop since it is continuously carried away by the cooling water, passing at a speed of $6 \mathrm{~mm} / \mathrm{ms}$.

Using lighter spheres would reduce the energy content in each sphere but would also increase the required target length. This would be detrimental to the phase space density of the pions in the collection system and will thus not be considered here.

A further reduction of the energy deposition from $14.5 \mathrm{~J} / \mathrm{g}$ to $11.3 \mathrm{~J} / \mathrm{g}$ can be achieved by enlarging slightly the width of the incident proton beam together with the target. An increase of the beam width of only about $15 \%(\mathrm{w}=1.32 \mathrm{~cm})$ would be necessary to accept the $4 \mathrm{MW}$ design beam intensity. One may also consider to shape a beam with a more uniform density, which reduces the energy density along the center while raising it slightly outside the center.

It may even be envisaged to use smaller spheres along the target center and increasing the size towards the rim. There the energy deposition density gets smaller and the conditions for the evacuation of the heat from these spheres are less demanding. 


\section{Stresses and Pressures in the Target}

\subsection{Static stresses in the spheres}

One of the interesting features of the granular target is that the instantaneous temperature rise induced by the beam is about uniform across each sphere since its diameter is small compared to the r.m.s. radius of the instantaneous temperature profile across the target. Thus, at least at the instant of the beam impact no static thermal stresses are created in the sphere. This temperature profile changes however, once heat diffusion through the sphere towards its cooled surface sets in.

With the thermal diffusivity $\delta$ of Ta

$$
\delta=\frac{\lambda}{c \rho}=21.6 \cdot 10^{-6} \mathrm{~m}^{2} / \mathrm{s}
$$

$\lambda$ : Thermal conductivity, $\lambda=55 \mathrm{~W} / \mathrm{m} \mathrm{K}$

the average duration over which heat diffuses from the center of the sphere with a radius of $\mathrm{R}=1 \mathrm{~mm}$ to the $\mathrm{rim}$ is

$$
\mathrm{t}_{\mathrm{d}} \approx 5 \mathrm{~ms}
$$

which is reasonably small compared to the time constant of the cooling at the surface.

Since the internal heat diffusion process is faster than the decrease of the temperature at the rim no substantial thermal gradient will build up inside the sphere, its temperature will decrease between the proton pulses about uniformly through its volume. Thus no substantial quasi static thermal stresses will occur inside the sphere.

Timoshenko [Ref. 5] quotes for a similar case a maximum transient stress $\sigma_{d}$ of

$$
\sigma_{\mathrm{d}} \approx 0.6 E \alpha \Delta \mathrm{T}_{0}
$$

E : Young's modulus, $E=16 \times 10^{10} \mathrm{~N} / \mathrm{m}^{2}$

$\alpha$ : Linear thermal expansion coefficient, $\alpha=6.5 \times 10^{-6} \mathrm{~K}^{-1}$

which would for $\Delta \mathrm{T}_{0}=75 \mathrm{~K}$ give a stress

$$
\sigma_{\mathrm{d}} \approx 47 \mathrm{MPa}
$$

which is well below the yield stress of high strength Tantalum of $\sigma_{0.2} \approx 1000 \mathrm{MPa}$. 


\subsection{Thermal shock in the spheres}

For an infinitely short proton pulse the heating of the spheres occurs instantly which gives rise to an instant uniform pressure in the " hottest " sphere of

$$
\begin{aligned}
& \mathrm{P}_{\mathrm{O}}=\frac{E \alpha \Delta T}{1-2 v} \\
& \mathrm{v}: \text { Poisson ratio; } v \approx 1 / 3
\end{aligned}
$$

which thereafter will oscillate with a characteristic time $t_{s}$ of

$$
\mathrm{t}_{\mathrm{s}} \approx R / \mathrm{s}
$$

$\mathrm{s}:$ Velocity of sound, $\mathrm{s} \approx 3.8 \times 10^{3} \mathrm{~m} / \mathrm{s}$

which yields

$$
\mathrm{t}_{\mathrm{s}} \approx 0.25 \mu \mathrm{s} \text {. }
$$

Since, however the envisaged duration of the proton pulse $\tau$ of $3.3 \mu \mathrm{s}$, over which each sphere is heated, is large compared to $t_{s}$, the dynamic stress $P_{o}$ is reduced by a factor of $\tau / t_{s} \approx 13$ [Ref. 6]

$$
\mathrm{P} \approx \mathrm{P}_{\mathrm{o}} \mathrm{t}_{\mathrm{s}} / \tau
$$

The dynamic behavior of free spheres can further be illustrated by considering the energy, which is stored in each sphere due to its compression by rapid heating and which might, at most, be transformed into kinetic energy.

This energy density $\mathrm{dE}_{\mathrm{c}} / \mathrm{dv}$ is given by

$$
\mathrm{dE}_{\mathrm{c}} / \mathrm{dv}=\frac{\left[\mathrm{P}(\mathrm{r}) \cdot \mathrm{t}_{\mathrm{s}} / \tau\right]^{2}}{3 \mathrm{E}}
$$

With a temperature increase of $100 \mathrm{~K}$ in the central, hottest sphere, the stored energy $\hat{E}_{c}$ is of the order of $10^{-6} \mathrm{~J}$ and integrated over all spheres, taking into account the radial Gaussian temperature and thus pressure distribution, yields at most

$$
\overline{\mathrm{E}}_{\mathrm{c}} \approx 0.1 \mathrm{~J} \text {. }
$$

This small amount of energy would for example be dissipated when the whole target would be dropped from a height of only $3 \mathrm{~mm}$. From this small impact, nevertheless some audible rattling and shaking will result, but should not lead to a rapid destruction of the target.

The individual spheres, although not completely free, will be in relatively loose contact. This is illustrated, for example, by the fact that the radius of the hottest sphere increases by $0.64 \mu \mathrm{m}$ over the duration of $3.3 \mu$ s of each proton pulse. 
This small displacement is of the same order or smaller than the roundness of precision spheres and their surface roughness. Still, the local contact among the spheres will lead to somewhat constrained spheres and to increase of pressure and thus to higher stored energy. In the extreme case, when no free surfaces are provided at all inside the target material, like in bulk Tantalum, the total stored kinetic energy is given by

$$
E_{c}=\int_{V} \frac{P^{2}(r)}{3 E} d v
$$

which would be of the order of

$$
\mathrm{E}_{\mathrm{c}}=17 \mathrm{~J} .
$$

The corresponding " drop height " would then be $0.48 \mathrm{~m}$.

The energy of $\mathrm{E}_{\mathrm{c}} \approx 0.1 \mathrm{~J}$ stored in a target, when made of free Ta-spheres as compared to $\mathrm{E}_{\mathrm{c}} \approx 17 \mathrm{~J}$ for a bulk target demonstrates the range within which this target might operate. Still, it is expected that the target is much closer to an ensemble of free spheres than to bulk material.

For comparison, the stored energy in bulk Mercury would be

$$
\mathrm{E}_{\mathrm{c}} \approx 600 \mathrm{~J}
$$

about 35 times more than in bulk Ta. This is due to the unfavourably high thermal expansion coefficient and the compressibility of $\mathrm{Hg}$. This illustrates that a solid, in particular when this solid consists of small spheres, responds much less energetically to the impact of the beam than a compressible liquid.

\subsection{Instantaneous pressure in the cooling water}

The instantaneous heating of the cooling water will lead to pressure waves, due to the thermal expansion of water which is initially prevented by mass inertia. This peak pressure in the water is of the order of

$$
\Delta \widehat{\mathrm{P}}_{\mathrm{W}} \approx \frac{\alpha_{\mathrm{W}} \cdot \Delta \mathrm{T}_{\mathrm{W}}}{\kappa}
$$

$\Delta \mathrm{T}_{\mathrm{W}}:$ Instantaneous temperature rise in water at the center of the target (scaled from Tantalum), $\Delta \mathrm{T}_{\mathrm{W}}=\mathrm{q}_{0} / \mathrm{c}_{\mathrm{W}}=3.6 \mathrm{~K}$

$c_{\mathrm{W}}: \quad$ Specific heat of water, $\mathrm{c}_{\mathrm{W}}=4 \times 10^{3} \mathrm{~J} / \mathrm{kg} \mathrm{K}$

$\alpha_{\mathrm{W}}$ : Volume expansion coefficient of water, $\alpha_{\mathrm{W}} \approx 10^{-3} \mathrm{~K}^{-1}$

$\kappa$ : $\quad$ Compressibility of water, $\kappa=5.6 \times 10^{-10} \mathrm{~m}^{2} / \mathrm{N}$. 
Preliminary computations with MARS have shown that the energy deposition per unit mass remains about constant even down to water, adjacent to the Ta-spheres [Ref. 2]. Therefore, with the above maximum energy density $\mathrm{q}_{0}=14.5 \mathrm{~J} / \mathrm{g}$ for the water it results

$$
\Delta \widehat{\mathrm{P}}_{\mathrm{W}}=6.4 \mathrm{MPa}(\sim 60 \text { bars })
$$

An additional compression of the water may come from the thermal expansion of the spheres which may add at most $5 \mathrm{MPa}$. However, it should be emphasized that the pressure waves transmitted from the spheres into the water have a much higher frequency $(\sim \mathrm{MHz})$ than those originating in the water itself $(\sim 10 \mathrm{kHz})$.

It is also questionable if waves in water propagate in the same way through the " forest " of spheres as in bulk water. Frictional energy loss between the water and the spheres will certainly damp the pressure wave emerging from the center and hitting the container wall. In all, a peak pressure of $10 \mathrm{MPa}$ at the container wall is thus considered to have a substantial safety margin.

\section{The Target Container}

\subsection{The target cylinder}

As discussed above the container wall will have to stand pressure amplitudes of the water of at most $10 \mathrm{MPa}$ at a frequency of about $10 \mathrm{kHz}$, which are induced by the proton bursts each $13.3 \mathrm{~ms}$. Titanium as cylinder material is suggested.

As the eigen frequency for radial oscillations of the Ti-pipe, given by

$$
\begin{gathered}
v_{\mathrm{Ti}}=\frac{\mathrm{s}_{\mathrm{Ti}}}{2 \pi \mathrm{R}} \\
\mathrm{s}_{\mathrm{Ti}}: \text { sound velocity in } \mathrm{Ti}, \mathrm{s}_{\mathrm{Ti}}=4.5 \times 10^{3} \mathrm{~m} / \mathrm{s}
\end{gathered}
$$

is about $30 \mathrm{kHz}$, it will respond to the pressure waves induced in the water at about $10 \mathrm{kHz}$.

This represents a major dynamic load and must be carefully considered. In order to limit the mechanical stresses in the Ti-cylinder, it must have a sufficient thickness. The circumferential stress $\sigma_{\varphi}$ and the axial stress $\sigma_{z}$ for a thin walled tube, closed at either end, are

$$
\begin{gathered}
\sigma_{\varphi}=\Delta \mathrm{P}_{\mathrm{W}} \cdot \mathrm{R} / \mathrm{d} \\
\sigma_{z}=\Delta \mathrm{P}_{\mathrm{W}} \cdot \mathrm{R} / 2 \mathrm{~d}
\end{gathered}
$$


and the von Mieses stress should be

$$
\sigma_{\mathrm{T}}<\Delta \mathrm{P}_{\mathrm{W}} \cdot \mathrm{R}_{/ \mathrm{d}} \approx 90 \mathrm{MPa}
$$

$\mathrm{R}$ : Target Radius, $\mathrm{R}=2.5 \mathrm{~cm}$

$\mathrm{d}$ : Wall thickness of the tube, $\mathrm{d}=0.25 \mathrm{~cm}$.

This is still a factor 5 - 10 below the static yield strength of a high quality Ti-alloy. Although for the cooling of this pipe, the surface to volume ratio of the cylinder wall is 7.5 times lower than for spheres, the energy deposition at the edge of the cascade (in particular since the container is made out of a light material, $\rho_{\mathrm{Ti}}=5 \times 10^{3} \mathrm{~kg} / \mathrm{m}^{3}$ ) is only a few percent of the value at the center. Therefore the cooling of the container wall should not cause a problem.

\subsection{The container windows}

The maximum energy deposition density $\mathrm{q}_{1}$ at the entrance window is about $60 \%$ of the maximum of $\mathrm{q}_{0}=14.5 \mathrm{~J} / \mathrm{g}$ pulse inside the target [Ref. 2]. Thus with $\mathrm{q}_{1}=8.6 \mathrm{~J} / \mathrm{g}$ and per pulse, the maximum instantaneous temperature rises per pulse for Titanium and Beryllium windows are

and

$$
\Delta \mathrm{T}_{1}, \mathrm{Ti}=\frac{\mathrm{q}_{1}}{\mathrm{c}_{\mathrm{Ti}}}=14.3 \mathrm{~K}
$$

$$
\Delta \mathrm{T}_{1}, \mathrm{Be}=\frac{\mathrm{q}_{1}}{\mathrm{c}_{\mathrm{Be}}}=4.5 \mathrm{~K}
$$

$\mathrm{c}_{\mathrm{Ti}}$ : Specific heat of Titanium, $\mathrm{c}_{\mathrm{Ti}}=0.6 \times 10^{3} \mathrm{~J} / \mathrm{kg} \mathrm{K}$

$\mathrm{c}_{\mathrm{Be}}$ : Specific heat of Beryllium, $\mathrm{c}_{\mathrm{Be}}=1.9 \times 10^{3} \mathrm{~J} / \mathrm{kg} \mathrm{K}$.

Quasi-static thermal stresses, induced by the radial Gaussian temperature profile from the beam as well as dynamic stresses induced by the rapid heating by the beam over $3.3 \mu$ s will occur. Each of them are of the order

$$
\sigma=\mathrm{E} \alpha \Delta \mathrm{T} \approx 10 \mathrm{MPa}
$$

which is still comfortably low.

In addition, the windows have to resist to pulsed pressures from the cooling water up to $10 \mathrm{MPa}$ which requires a sufficient thickness. On the other hand the windows should be kept as thin as possible to minimise the beam energy deposited in the window and to be removed by the water cooling from only one side. Spherical shells 
provide the highest pressure resistance at minimum thickness. The stress $\sigma$ due to the internal pressure $\mathrm{P}$ is :

$$
\sigma=\mathrm{P} \cdot \mathrm{R} / 2 \mathrm{~d}
$$

For a thickness of $\mathrm{d}=1 \mathrm{~mm}$ a stress of

$$
\sigma=125 \mathrm{MPa}
$$

arises which is considered as acceptable for both materials.

The time constant $t_{0}$ for the cooling of the window becomes

$$
t_{0}=\frac{c \cdot m}{\gamma \cdot F}=\frac{c \cdot \rho \cdot d}{\gamma}
$$

and yields for both metals about $\mathrm{t}_{0} \approx 150 \mathrm{~ms}$, about four times longer than for the cooling of the spheres with $\mathrm{t}_{0}=38 \mathrm{~ms}$. This is mainly due to the lower cooling surface to volume ratio for " disks ", like the windows.

With this time constant for the cooling of the windows the minimum temperature $T_{1}$ relative to the pulsed temperature rise $\Delta \mathrm{T}$ is

$$
\mathrm{T}_{1} \approx 11 \cdot \Delta \mathrm{T}
$$

and the temperature between which the windows would oscillate in " the steady state " is

$$
\begin{aligned}
& \text { Ti : } \quad \mathrm{T}=157^{\circ} \mathrm{C} \ldots 172{ }^{\circ} \mathrm{C} \\
& \text { Be : } \quad \mathrm{T}=50^{\circ} \mathrm{C} \ldots 55^{\circ} \mathrm{C}
\end{aligned}
$$

This demonstrates that the windows must carefully be optimized in terms of cooling, appropriate material, shape and thickness.

\section{Cooling with Liquid Metal}

\subsection{Cooling efficiency of liquid metal}

Clearly, to increase the cooling efficiency the use of liquid metals should be considered. These technologies have widely been developed in nuclear reactor research, including their highly radioactive environment. The operation of liquid metal cooled devices in a pulsed regime needs however to be investigated further.

Obvious candidates are liqu. Lithium $\left(\rho \approx 0.5 \times 10^{3} \mathrm{~kg} / \mathrm{m}^{3}\right.$, melting point $\left.180{ }^{\circ} \mathrm{C}\right)$ and Na-K-alloys $\left(\rho \approx 0.8 \times 10^{3} \mathrm{~kg} / \mathrm{m}^{3}\right.$, liquid at room temperature). Although Li may be of 
interest because of its low density and high specific heat which would cause low instantaneous temperature rises $\left(\Delta \mathrm{T}_{0}=3.6 \mathrm{~K}\right)$ and pressures but its relative high melting point would require an elevated average operating temperature of well above $200{ }^{\circ} \mathrm{C}$. Therefore only Na-K is considered at this stage, since its density and thermal quality are sufficient to handle the design energy deposition density at a relatively low average temperature.

With the peak energy deposition density from the design beam an instantaneous temperature rise in the " hottest " Ta sphere of $96 \mathrm{~K}$ is reached.

To estimate the cooling efficiency with $\mathrm{Na}-\mathrm{K}$ we proceed as before. For the heat transfer coefficient in the turbulent regime with a velocity of $6 \mathrm{~m} / \mathrm{s}$ between the spheres (The same liquid flow rate as for water is assumed) a value of at least

$$
\gamma \approx 120 \mathrm{~kW} / \mathrm{m}^{2} \mathrm{~K}
$$

can be expected. This implies a Nusselt number of about 10 which is very conservative and leads to a $\gamma$ which is at least 5 times larger than for water. The main reason is the high thermal conductivity of $\mathrm{Na}-\mathrm{K}$ of

$$
\lambda=24 \mathrm{~W} / \mathrm{mK}
$$

which is about 40 times larger than water.

Still, limited wetting of the Tantalum spheres by the Na-K-alloy and possible thermal surface barriers, created by chemical reactions on the spheres at elevated temperatures are of concern and must be investigated.

The high heat transfer coefficient should nevertheless lead to very fast cooling of the Ta-spheres between the proton pulses. The time constant $t_{0}$ for spheres with radius of $1 \mathrm{~mm}$ becomes

and the ratio

$$
\mathrm{t}_{0} \approx 7 \mathrm{~ms}
$$

$$
\Delta \mathrm{T}_{0} / \mathrm{T}_{1}=5.7
$$

With $\Delta \mathrm{T}_{0}=96 \mathrm{~K}$ the lowest temperature rise $\mathrm{T}_{1}$ of the sphere above the average of the cooling fluid just before a proton pulse is

$$
\mathrm{T}_{1}=17 \mathrm{~K} \text {. }
$$

The average temperature rise in the cooling medium during its passage is

$$
\Delta \mathrm{T}=\mathrm{T}_{\text {out }}-\mathrm{T}_{\text {in }} \approx 90 \mathrm{~K}
$$

at the same volume flow $\mathrm{V}$ as for water :

$$
\mathrm{V}=111 / \mathrm{s} \text {. }
$$


This temperature rise is well above that of water, mainly due to the lower specific heat of $\mathrm{Na}-\mathrm{K}$

$$
\mathrm{c}=10^{3} \mathrm{~J} / \mathrm{kg} \mathrm{K}
$$

Therefore an offset in temperature of the cooling medium, when it reaches the target center of about $45 \mathrm{~K}$ plus the inlet temperature of about $20^{\circ} \mathrm{C}$ have to be taken into account. The " hottest " sphere will thus oscillate between

$$
82^{\circ} \mathrm{C} \text { and } 178^{\circ} \mathrm{C} \text {. }
$$

This can be put into perspective with an " ideal " cooling with liquid Li, where the temperature of the hottest sphere would at best cycle between $200{ }^{\circ} \mathrm{C}$ and $296^{\circ} \mathrm{C}$.

\subsection{Temperature and pressure pulses in the liquid metal}

Assuming, as before the same peak energy deposition density of $14.5 \mathrm{~J} / \mathrm{g}$, independent of the material, an instantaneous peak temperature $\Delta \mathrm{T}$ in the Na-K-alloy of

$$
\Delta \mathrm{T}=14.5 \mathrm{~K}
$$

occurs. This again may lead to dynamic pressure amplitudes of

$$
\Delta \mathrm{P} \approx 30 \mathrm{MPa}(300 \text { bars })
$$

with

$\kappa:$ Compressibility of $\mathrm{Na}-\mathrm{K} \approx 2 \times 10^{-10} \mathrm{~m}^{2} / \mathrm{N}$

$\alpha_{\mathrm{v}}$ : Volume expansion coefficient, $\alpha_{\mathrm{v}} \approx 4.3 \times 10^{-4} \mathrm{~K}^{-1}$.

We note that due to the smaller compressibility of Na-K, combined with a larger $\Delta \mathrm{T}$ than in water, higher pressure amplitudes will result. This will solicit more the container walls and end caps and their thicknesses must be designed accordingly. Also material erosion at the solid/liquid interface at elevated pressures may be a subject of concern. However, as stated above, one may expect damping of the pressure wave during its propagation through the granular medium of the Ta-spheres protecting the container wall to a certain extent.

From this point of view, Lithium with a density of $500 \mathrm{~kg} / \mathrm{m}^{3}$ and a specific heat of $4 \times 10^{3} \mathrm{~J} / \mathrm{kg} \mathrm{K}$ (about the same as water) is more favourable since the instantaneous temperature $\Delta \mathrm{T}$ is only

$$
\Delta \mathrm{T}=3.6 \mathrm{~K}
$$

which should lead to lower pressure amplitudes. 
Increasing the thickness of the windows for better pressure resistance leads to higher average temperatures. This might be counteracted by cooling it from both sides, using a double-walled window, one thick one to resist the pressure waves from the bulk of the target and a second one to contain the cooling liquid and pressure initiated locally therein. One may also use locally lighter spheres close to the windows (see Fig. 1) so that at least the average temperatures at this location is diminished.

\section{Conclusion and Recommendation}

A stationary target, made out of small spheres, confined in a metal container and cooled by a fluid passing through the voids between the spheres has been assessed. As cooling fluid, initially water should be considered. Low density liquid metals may also be of interest. Efficient heat removal and low dynamic stresses and pressures are achieved, mainly due to the small size in the millimeter range of the target constituents in combination with relatively long proton bursts of several micro-second duration. By further computational and experimental studies the performance limits must be investigated. These studies must include detailed considerations of the lifetime, due to the fatigue induced by the very high rate of 6.5 Mio. cycles per day, of the target spheres and, in particular, of the entrance and exit windows. The lifetime must be balanced against the cost and effort to exchange the target in regular intervals. Dedicated laboratory tests without the need of a proton beam should be devised to elucidate these problems.

Energy deposition densities of the order of 15 to $20 \mathrm{~J} / \mathrm{g}$ and average power densities of $1 \mathrm{~kW} / \mathrm{g}$ or above may be within reach. This would comply with the CERN-NeutrinoFactory proposal with a beam of $4 \mathrm{MW}$ power at $2.2 \mathrm{GeV} / \mathrm{c}$, pulsed at $75 \mathrm{~Hz}$.

\section{Acknowledgment}

Thanks are due to S. Gilardoni who provided the input data from the MARS-cascade simulation code. This work greatly profited from guidance and stimulating discussions with B. Autin and P. Pugnat. 


\section{References}

[1] H. Haseroth, for the CERN Neutrino Factory Working Group at CERN, NuFact Note 45, " CERN Ideas and Plans for a Neutrino Factory " 2000.

[2] S. Gilardoni, CERN-PS, private communication, 2001.

[3] N. V. Mokhov, " The MARS Code System User's Guide ", Fermilab-FN-628, 1995.

N. V. Mokhov, " MARS Code Developments, Benchmarking and Applications ", Fermilab-Conf-00-066, 2000.

N. V. Mokhov, S. I. Striganov, A. Van Ginneken, S. G. Mashnik, A. J. Sierk and J. Ranft, " MARS Code Developments ", Fermilab-Conf-98/379, 1998; LANL Report LA-UR-98-5716, 1998; Nucl-th/9812038 v2, 16 Dec. 1998.

O. E. Krivosheev and N. V. Mokhov, " A New MARS and its Applications ", Fermilab-Conf-98/43, 1998.

[4] G. Linder et al., Mikrowärmeübertrager und Mikroreaktoren, Symposion on Micro-Technique ACHEMA, Frankfurt, Germany, 1994.

[5] S. P. Timoshenko and J. N. Goodier, " Theory of Elasticity ", Mc Graw-Hill Book Company, Third Edition, 1973.

[6] P. Pugnat and P. Sievers, " Thermally induced waves in cylinders and spheres ". To be published. 


\section{GRANULAR TARGET \\ COOLED BY LIQUID}
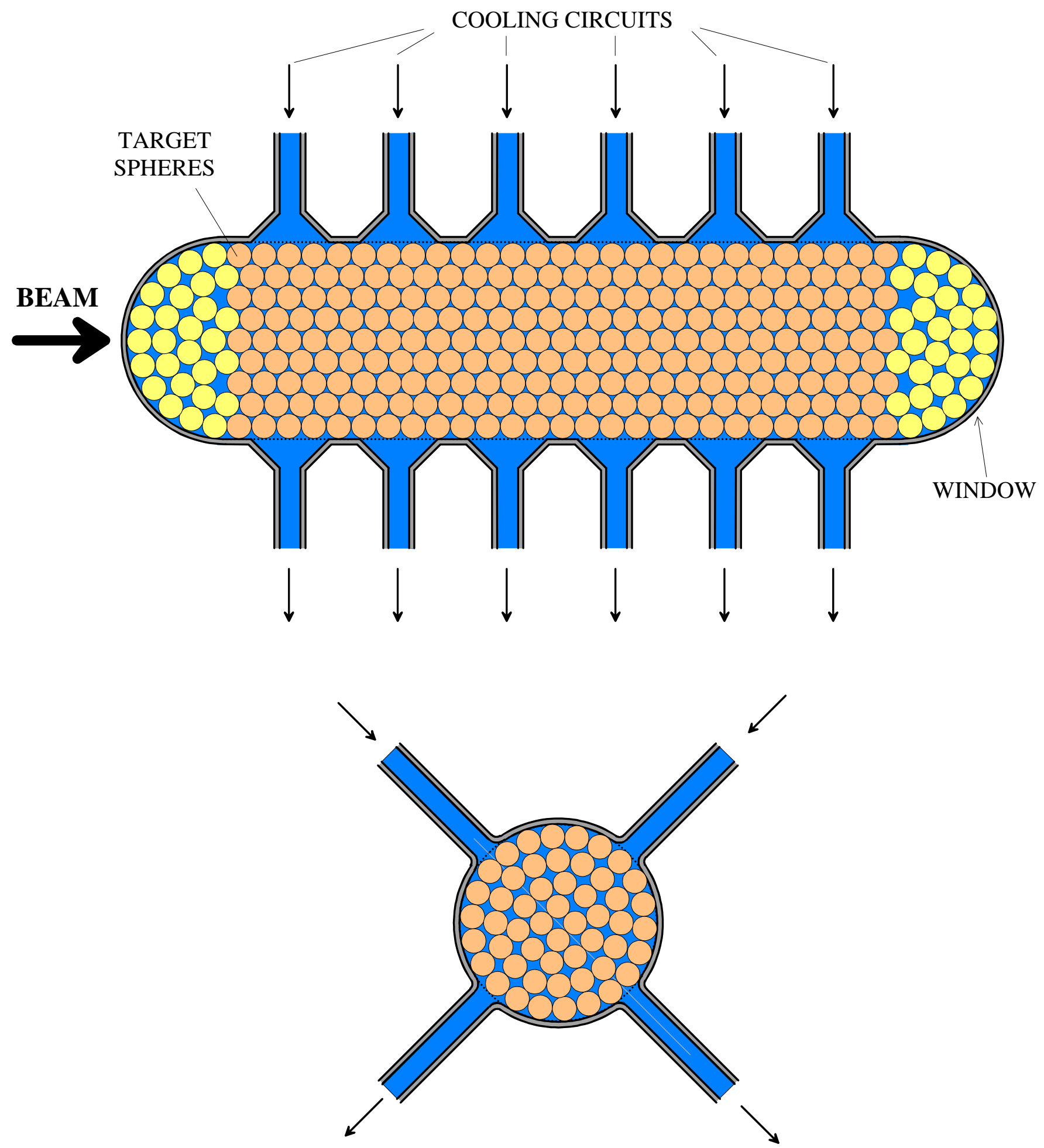

Fig. 1 : Principle lay-out of a liquid cooled, granular target. Tantalum spheres with a diameter of about $2 \mathrm{~mm}$ are confined in a Titanium container and cooled by water (or possibly liquid metal) traversing the voids between the spheres. 